\title{
A STUDY OF THE MINIMUM WALL THICKNESS FOR AN EXTRUDED ALUMINUM VACUUM CHAMBER*
}

\author{
Emil Trakhtenberg, Greg Wiemerslage, Argonne National Laboratory, Argonne, IL 60439, U.S.A.
}

\section{Abstract}

Decreasing an ID vacuum chamber wall thickness is a way to achieve a smaller ID gap and a higher magnetic field without decreasing the clear beam aperture of the chamber. Multiple extruded aluminum ID vacuum chambers with 1-mm wall thickness were developed and fabricated at Argonne for the APS and several other synchrotron radiation facilities [1]. Thinner walls have been avoided due to fear that porosity and defects from the extrusion process would result in vacuum leaks. There were also concerns that thinner walls may have excessive deformation or may not withstand the stresses.

Recently, several extrusions have been machined to a wall thickness of less than $1 \mathrm{~mm}$ to determine the practical limits. Using the extrusion for the insertion device vacuum chamber (ID VC) for the DESY FEL project with a $9.5-\mathrm{mm}$ inner diameter and the LCLS test vacuum chamber extrusion, we decreased the wall thickness to $0.6,0.5$, and $0.4 \mathrm{~mm}$ to test the vacuum integrity for a thin wall in these extrusions. Structural analysis and test results are presented.

\section{EXPERIMENTAL REASONINGS}

Fourth-generation synchrotron radiation source (SRS) projects are based mostly on the Free-electron laser (FEL) concept such as TESLA, LCLS, and FERMI to name a few. Key components of all these projects are long undulator lines with permanent magnets and a rather small pole gap to achieve the maximum peak field. There are many possible design options for the vacuum chambers for such undulators, and one of them is to use aluminum extrusions and precisely machine an area for the undulator gap.

Such an extrusion was used for the TESLA Test Facility where the nominal aperture is $9.5 \mathrm{~mm}$ and the nominal wall thickness is $1 \mathrm{~mm}$. The standard vacuum chambers for the APS undulators are also fabricated from an aluminum extrusion and also have a nominal wall thickness of $1 \pm 0.1 \mathrm{~mm}$.

During the extrusion process, a billet of 6063 alloy of aluminum is heated to a temperature ranging from 400 to 480 degrees Centigrade and then pressed through a steel die by a hydraulic ram that uses anywhere from 100 tons to 15,000 tons of force. The billet first passes through a steel mandrel section that defines the interior geometry of the chamber. The interior geometry has to be supported and positioned by steel supports to the outside diameter of the mandrel. The billet "breaks" as it passes those

\footnotetext{
* Work at Argonne National Laboratory is supported by the U.S. Department of Energy, Office of Science, Office of Basic Energy Sciences under Contract \# DE-AC02-06CH11357.
}

supports. As the billet then passes through the steel cap section, which forms the outside shape of the chamber, the voids created by the supports of the inner shape defined by the mandrel then get closed as the aluminum material rejoins. Therefore, one has to be sure that an extruded aluminum chamber is vacuum-tight even when the wall thickness is small (see Fig. 1). Although we have never experienced leaks through the 1-mm wall of any of the chambers we have produced, we have now tested the possibility of decreasing the wall thickness down to at least $0.5 \mathrm{~mm}$.
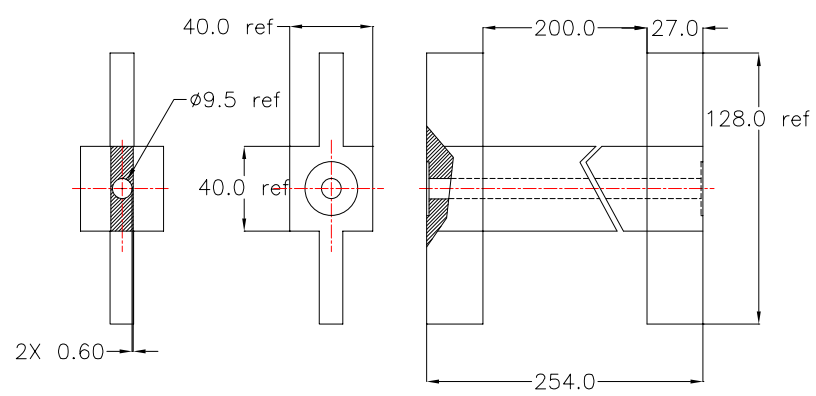

Figure 1: Extrusion test piece.

\section{EXPERIMENTAL PARAMETERS}

For our first experiments we used the extrusion for the vacuum chamber for the TESLA Test Facility and machined as it shown in Fig. 1. We initially machined the wall thickness to $0.6 \mathrm{~mm}$. After machining, the part was plugged by welding from the right side. A bi-metal transition with a 1.33 " ConFlat flange was welded on the left side (see Fig. 2).

The wall thickness was measured mechanically during machining and also ultrasonically after the machining process. The results of the two measurement techniques were consistent.

The wall thickness was then decreased in two steps to 0.5 and $0.4 \mathrm{~mm}$. Vacuum inside the first sample at $0.4 \mathrm{~mm}$ was $5.6 \cdot 10^{-5}$ Torr and $2.7 \cdot 10^{-5}$ Torr for the second sample at $0.4 \mathrm{~mm}$ (samples were not baked). After baking the second sample to 150 degrees Centigrade for twenty-four hours, the vacuum was improved to $1.4 \cdot 10^{-7}$ Torr with a $0.4 \mathrm{~mm}$ wall thickness. For all tests we used an AlcatelASM 180 leak detector. There were no leaks detected in any of these tests. The wall deflection was measured by running a dial indicator across the surface of the thin area. We used a Mitutoyo model number 513-203 dial indicator with 0.0001 " graduations that was last calibrated on $3 / 5 / 07$. There was no wall deflection detected during these tests. 
The same types of tests were performed for the actual LCLS vacuum chamber probe extrusion (see Fig. 3). The wall thickness was machined initially to $0.6 \mathrm{~mm}$, then to $0.5 \mathrm{~mm}$, and then to $0.4 \mathrm{~mm}$. Vacuum before baking was $2.1 \cdot 10^{-7}$ Torr during the $0.6 \mathrm{~mm}$ test, $1.7 \cdot 10^{-7}$ Torr during the $0.5 \mathrm{~mm}$ test, and $1.3 \cdot 10^{-7}$ Torr for the $0.4 \mathrm{~mm}$ test. There were no leaks detected in any of the tests. The tests also showed no detectable deflection of the thin wall, which was consistent with the preliminary calculations (see Fig. 4 and Fig. 5).

It is important to note that the extrusion for the TESLA test was made by Taber Metals and the extrusion for the LCLS sample was made by a different vendor, Cardinal Aluminum Co. This lends credibility to the fact that regardless of the vendor, the extrusion process yields chambers that can be machined to a thin wall without porosity.

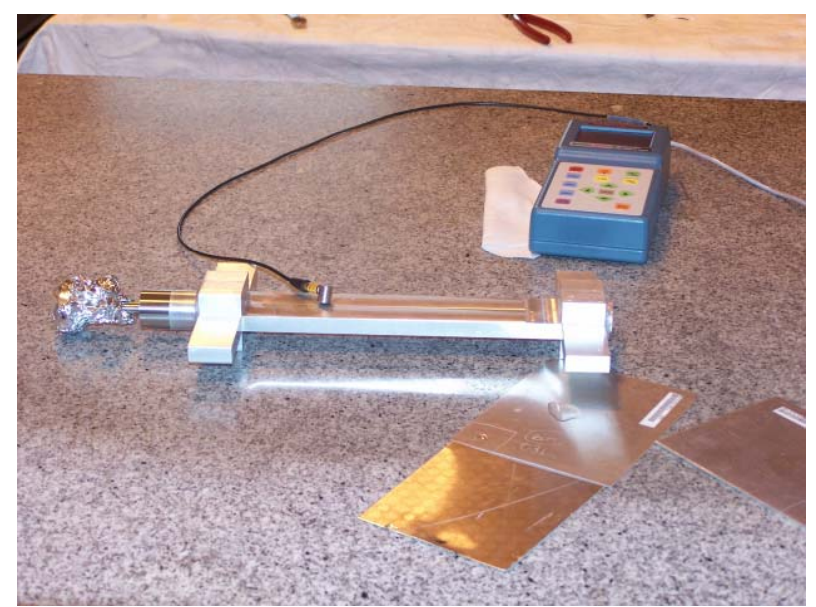

Figure 2: Test model of the DESY VC extrusion shown with the ultrasonic thickness gauge and calibration plates.

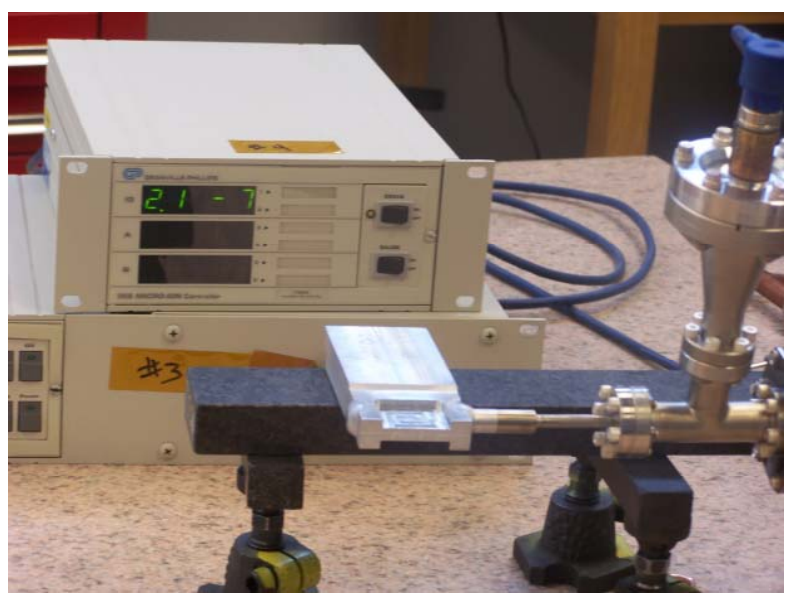

Figure 3: Experimental set up of the LCLS sample extrusion machined to $0.5-\mathrm{mm}$ wall thickness under vacuum.

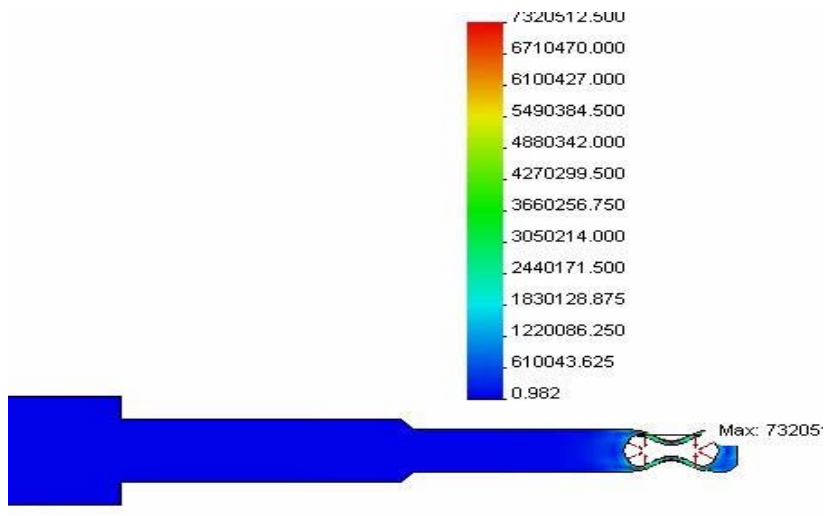

Figure 4: The calculated maximum stress is $73.2 \mathrm{~kg} / \mathrm{cm}^{2}$ for the extruded LCLS chamber with a $0.5-\mathrm{mm}$ wall thickness.

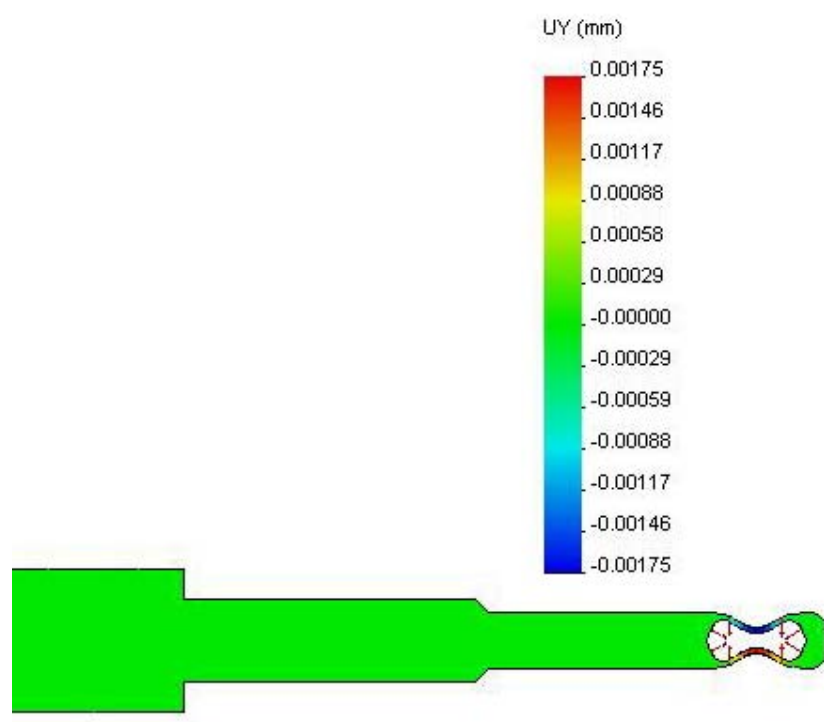

Figure 5: The calculated maximum deflection is 1.75 microns for the extruded LCLS chamber with a $0.5-\mathrm{mm}$ wall thickness.

\section{CONCLUSION}

The results clearly show that the vacuum integrity of the extruded aluminum alloy 6063-T5 allows the manufacture of extruded aluminum vacuum chambers with a wall thickness of $0.5 \mathrm{~mm}$. In the future we will test full-length extrusions and different chamber profiles.

\section{REFERENCES}

[1] E. Trakhtenberg, G. Wiemerslage, P. Den Hartog, "New insertion device vacuum chambers at the Advanced Photon Source," PAC 2003, Portland, OR May 2003, http://www.jacow.org. 